\title{
Anesthetic management of a chronic liver disease patient with very low platelet counts by considering a rebalanced hemostasis - A case report -
}

Received February 14, 2018

Revised 1st, July 2, 2018 2nd, July 12, 2018

Accepted July 13, 2018

\section{Corresponding author}

Gaab-Soo Kim, M.D., Ph.D.

Department of Anesthesiology and

Pain Medicine, Samsung Medical

Center, Sungkyunkwan University

School of Medicine, 81 Irwon-ro,

Gangnam-gu, Seoul 06351, Korea

Tel: 82-2-3410-0360

Fax: 82-2-3410-0361

E-mail: gskim@skku.edu

ORCID

http://orcid.org/0000-0002-9383-2652

\author{
Bobae Han, Suk Young Lee, and Gaab-Soo Kim
}

Department of Anesthesiology and Pain Medicine, Samsung Medical Center, Sungkyunkwan University School of Medicine, Seoul, Korea

The coagulation profile of patients with end-stage liver disease (ESLD) is different from that of healthy individuals. Because hemostasis is rebalanced in chronic liver disease, prophylactic transfusion of blood products may be not necessary for these patients even if they show severe coagulation dysfunction in conventional coagulation results. A 44-year-old man with hepatocellular carcinoma, cholangiocarcinoma and liver cirrhosis was scheduled for extra-hepatic mass excision under general anesthesia. His preoperative tests showed severe thrombocytopenia $19 \times 10^{9} / \mathrm{L}$. The patient underwent extrahepatic mass excision surgery under general anesthesia without transfusion of blood products. The post-operative course was uneventful without requiring any further hemostatic therapy. In this case report, we focus on the concept of rebalanced hemostasis in ESLD, and coagulation management based on rotational thromboelastometry.

Keywords: Blood coagulation; Blood platelets; Rebalanced hemostasis; Rotational thromboelastometry; Thromboelastography.
Liver disease affects many organ systems including the hemostatic system $[1,2]$. Moreover, impaired hepatic synthesis of thrombopoietin, splenic sequestration, and rapid turnover of thrombocytes often cause thrombocytopenia in end-stage liver disease (ESLD) patients. Because of this, in the past, patients with cirrhosis have been thought of as in an "autoanticoagulated state." However, because there is a relative deficiency of both pro-coagulant and anti-coagulant factors in ESLD patients, this classical interpretation has been replaced by the concept of "rebalanced hemostasis." This concept was firstly mentioned by Lisman and Porte [3] in 2010. According to this concept, the hemostatic alterations in chronic liver disease result in a new balance. However, this balance is much more fragile and can shift easily toward either bleeding or thrombosis $[3,4]$. Assessment of the risk of bleeding in ESLD patient is not easy by conventional coagulation tests due to their inherent limitations. Viscoelastic tests such as thromboelastography (TEG) or rotational thromboelastometry (ROTEM) provide information on the overall coagulation system and therefore, may be better tools to gauge the risk of bleeding than conventional coagulation tests [4]. We report a case of a patient who had severe thrombocytopenia $\left(19 \times 10^{9} / \mathrm{L}\right)$ due to ESLD but underwent major surgery without any intraoperative and postoperative transfusions.

\section{CASE REPORT}

A 44-year-old man (body weight $73.9 \mathrm{~kg}$, height $174.6 \mathrm{~cm}$, type $\mathrm{O}$ blood) with combined hepatocellular carcinoma (HCC) and cholangiocarcinoma with liver cirrhosis was ad-

This is an Open Access article distributed under the terms of the Creative Commons Attribution Non-Commercial License (http://creativecommons.org/licenses/by-nc/4.0) which permits unrestricted non-commercial use, distribution, and reproduction in any medium, provided the original work is properly cited. 
mitted for laparoscopic mass excision. Computed tomography showed splenomegaly and an extrahepatic- mass, which was about $2.8 \mathrm{~cm}$ in diameter and located in the perihepatic space, adjacent to liver segment 6 (Fig. 1). The patient's Child-Pugh class was B with a score of 7 . He had been treated with a transarterial chemoembolization procedure three times and had underwent a wedge resection and lobectomy for lung metastasis of HCC. The preoperative laboratory value showed a hemoglobin level of $11.8 \mathrm{~g} / \mathrm{dl}$, hematocrit of $34 \%$, platelet count of $19 \times 10^{9} / \mathrm{L}$, prothrombin time (PT) of 19.3 seconds with an international normalized ratio (INR) of 1.60, and activated partial thromboplastin time (aPTT) of 44.1 seconds. Other laboratory tests including pulmonary function test showed normal findings. After discussing with the surgeon, it was decided that the patient would not receive prophylactic platelet transfusion despite the very low platelet count and to do a transfusion during surgery if necessary.

Electrocardiography, pulse oximetry and noninvasive blood pressure were applied before induction, and the initial vital signs showed a blood pressure 120/70 $\mathrm{mmHg}$, a heart rate of 90 beats $/ \mathrm{min}$, and a pulse oximeter saturation of $100 \%$. Anesthesia was induced with intravenous thiopental sodium $350 \mathrm{mg}$ and vecuronium $10 \mathrm{mg}$ and maintained with sevoflurane. Airway maintenance was done with a laryngeal mask airway (Protector, Teleflex Medical, Japan). Right radial

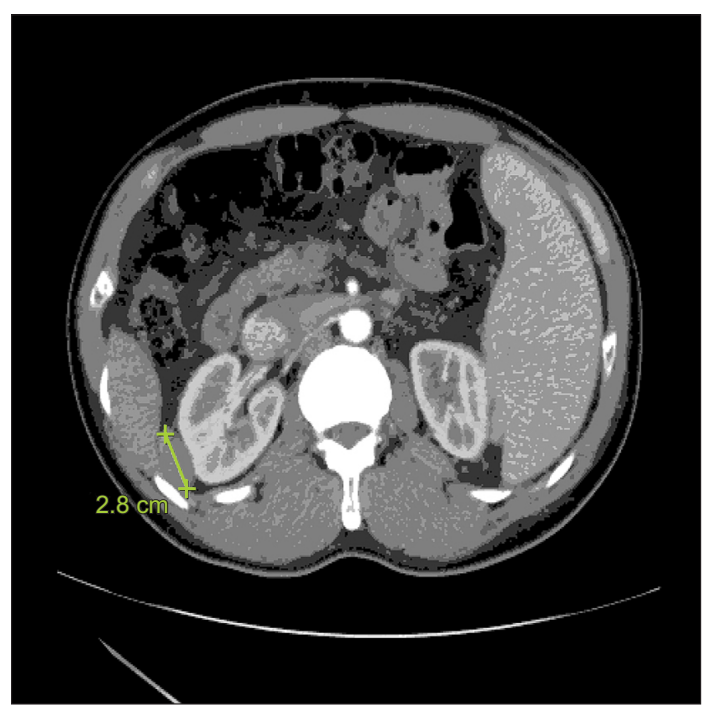

Fig. 1. Computed tomography (CT) of the the extrahepatic mass. CT shows a slight increase in the size $(2.8 \mathrm{~cm})$ of the presumed seeding nodule in the peri-hepatic space, adjacent to liver segment 6 with splenomegaly. artery was cannulated, and there were no hemodynamic instabilities during the induction. After the induction, tests for the platelet count, the plasma von Willebrand factor (vWF) level, and "real time" coagulation status using the rotational thromboelastometry (ROTEM Analyzer, Tem International $\mathrm{GmbH}$, Germany) were done. The platelet count was $24 \times$ $10^{9} / \mathrm{L}$ and, the plasma vWF antigen level was $179 \%$ (reference range: $52-155 \%$ for blood group O). The ROTEM (NATEM) showed a clotting time (CT) of 683 seconds (reference range: 300-1,000 seconds), a clot formation time (CFT) of 716 seconds (reference range: $150-700$ seconds), an alpha angle of $31^{\circ}$ (reference range: $30-70^{\circ}$ ), a maximum clot firmness (MCF) of $26 \mathrm{~mm}$ (reference range: $40-65 \mathrm{~mm}$ ), and a LI60 of 91\% (reference range: $76-96 \%$ ).

After placing the surgical retractor, we changed the anesthetic gas from sevoflurane to isoflurane. Vital signs and other monitoring parameters including end-tidal carbon dioxide, lactate, and electrolytes were within normal range. The procedure was converted from laparoscopic to open due to adhesions around the right kidney, but the patient did not show any sign of bleeding tendency. The total anesthesia time was 2 hours and 21 minutes, and only $700 \mathrm{ml}$ of Plasma Solution $A^{\circledR}$ (CJ HealthCare Corp., Korea) were intravenously administered. The estimated blood loss was $270 \mathrm{ml}$, and total urine output was $50 \mathrm{ml}$. We assessed the patient's coagulation status using complete blood count and ROTEM (NATEM) tests at the end of the surgery. The results were a hemoglobin level of $12.1 \mathrm{~g} / \mathrm{dl}$, a hematocrit level of $36 \%$, and a platelet count of $24 \times 10^{9} / \mathrm{L}$. The ROTEM values were similar to the initial values, with a CT of 698 seconds, a CFT of 439 seconds, an alpha angle of $38^{\circ}$, a MCF of $32 \mathrm{~mm}$, and a LI60 of 93\% (Table 1).

The patient was transferred to the post-anesthesia care unit after surgery and was discharged on the 6th postoperative day (POD) without any complications and transfusions. The antithrombin III value was $68 \%$ (reference range: $83-123 \%$ ) on the 80th POD.

\section{DISCUSSION}

Chronic liver disease results in complex alterations of all phases of hemostasis, and the net effect is considered to be a rebalanced hemostasis [3,4]. Deficiencies in pro-coagulant proteins are balanced by deficiencies in anti-coagulant proteins such as protein $\mathrm{C}$ and $\mathrm{S}$ and anti-thrombin [3]. Throm- 
Table 1. Flow Chart of the Coagulation Tests

\begin{tabular}{lccccccc}
\hline \multicolumn{1}{c}{ Time of sampling } & Platelet $(\mathrm{L})$ & CT $(\mathrm{s})$ & CFT $(\mathrm{s})$ & $\alpha\left(^{\circ}\right)$ & MCF $(\mathrm{mm})$ & LI60 (\%) & vWF (\%) \\
\hline Preoperative 1 day & $19 \times 10^{9}$ & - & - & - & - & - \\
After induction (8:25 a.m.) & $24 \times 10^{9}$ & 683 & 716 & 31 & 26 & 91 & 179 \\
End of operation (10:20 a.m.) & $24 \times 10^{9}$ & 698 & 439 & 38 & 32 & 93 & - \\
Postoperative 1 day (8:00 a.m.) & $24 \times 10^{9}$ & - & - & - & - & - & - \\
\hline
\end{tabular}

Platelet (reference range: $141-316 \times 10^{9} / \mathrm{L}$ ), CT: clotting time (reference range: 300-1,000 s), CFT: clot formation time (reference range: $150-700$ s), $\alpha$ : alpha angle (reference range: $30-70^{\circ}$ ), MCF: maximum clot firmness (reference range: $40-65 \mathrm{~mm}$ ), LI60: lysis index 60 (reference range: 76-96\%) and vWF: von Willebrand factor (reference range: 52-155\% ).

bocytopenia, a typical feature of chronic liver disease, is balanced by increased platelet function with high levels of vWF and low levels of ADAMTS 13 which cleaves vWF $[3,4]$. The fibrinolytic system may also be rebalanced by the concomitant decrease of antifibrinolytics (antiplasmin and thrombin activatable fibrinolysis inhibitor) and plasminogen $[4,5]$. In this case, the patient showed a rebalanced hemostasis: The decrease in pro-coagulant levels (increased PT) was balanced by a decrease in antithrombin III levels, and the low platelet count was balanced by increased levels of vWF and no hyperfibrinolysis on the ROTEM.

Conventional coagulation tests do not fully reflect the derangement in hemostasis in ESLD patients. For example, PT and aPTT tests are not sensitive to the deficiency of anticoagulants. The PT test only assesses the function of a discrete number of pro-coagulant proteins (factors VII, X, V, and II and fibrinogen) and therefore cannot reflect the true hemostatic status of a patient because it does not consider the function of the protein $\mathrm{C}$ and $\mathrm{S}$ pathway, antithrombin, and tissue factor pathway inhibitor $[4,5]$. Thus, moving away from the traditional laboratory measures of coagulation such as PT, aPTT and platelet counts to newer functional viscoelastic measures of coagulation such as TEG or ROTEM may be better for assessing coagulopathy in patients with chronic liver disease who undergo surgery [4-6]. TEG or ROTEM helps identify different coagulation defects including hyperfibrinolysis, hypofibrinogenemia, thrombocytopenia and hypercoagulopathy. These tests are point-of-care coagulation monitoring systems that rapidly evaluate the viscoelasticity of the whole blood, enabling the entire clotting process, from clot initiation and formation to clot stability, to be assessed [7-9].

Prophylactic platelet transfusion for patients having major elective non-neuraxial surgery is recommended if the platelet counts are less than $50 \times 10^{9} / \mathrm{L}$. For neurosurgery, platelet transfusion is recommended if the platelet counts are less

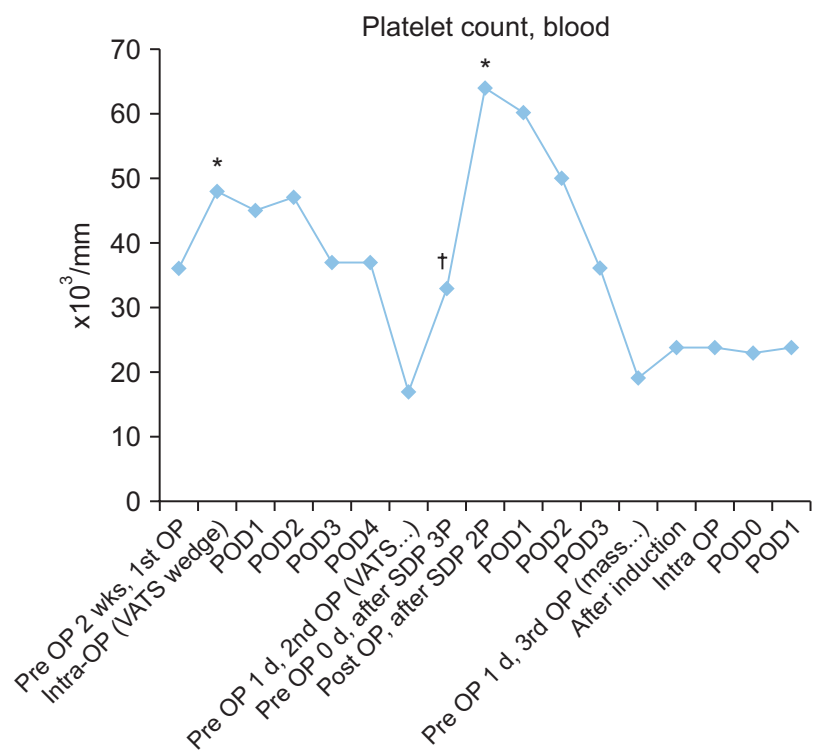

Fig. 2. Variation in the platelet counts after transfusion of single donor platelets (SDP). OP: operation, POD: postoperative day. *Platelet counts after 2 units of SDP transfusion. ${ }^{\dagger}$ Platelet counts after 3 units of SDP transfusion.

than $10 \times 10^{10} / \mathrm{L}[10]$. However, this recommendation should be applied with caution in ESLD because of the rebalanced hemostasis. Actually, patients undergoing liver transplantation in our hospital receive single donor platelets (SDP) only when the platelet count is below $30 \times 10^{9} / \mathrm{L}$. In this case, we did not perform any prophylactic transfusion even though the laboratory results revealed severe coagulopathy with a PT of 19.3 seconds, an INR of 1.60, an aPTT of 44.1 seconds, and a platelet count of $19 \times 10^{9} / \mathrm{L}$. The patient had previously undergone lung surgery twice, and had showed similar preoperative conventional coagulation tests such as the PT, aPTT, and platelet counts. In the previous two major surgeries, two and five units of SDP were administered before or during surgery, respectively, and the platelet counts increased to only about $10 \times 10^{9} / \mathrm{L}$ with 1 unit of SDP, and the final platelet counts were below the normal range in both cases (Fig. 2). 
However, the estimated blood loss was only 100 and $200 \mathrm{ml}$ during the first and second surgeries, respectively, and there was no postoperative bleeding complication in both surgeries. From this, we had expected that the patient might have a rebalanced hemostasis. We closely observed the bleeding tendency in the surgical field and performed ROTEM tests to determine the patient's actual coagulation status instead of relying on the conventional coagulation tests. The ROTEM result during surgery revealed a near normal coagulation status, and reflected the actual bleeding tendency in the surgical field better than the conventional coagulation tests.

In conclusion, if an ESLD patient is suspected of having a rebalanced hemostasis, ROTEM can be a more useful coagulation monitoring tool than the conventional coagulation tests. Moreover, it may be helpful to use ROTEM when making decisions about prophylactic transfusions.

\section{REFERENCES}

1. Shah NL, Northup PG, Caldwell SH. A clinical survey of bleeding, thrombosis, and blood product use in decompensated cirrhosis patients. Ann Hepatol 2012; 11: 686-90.

2. Shah NL, Caldwell SH. Assessing the risk of bleeding and clotting in cirrhosis. Clin Liver Dis 2016; 7: 26-8.
3. Lisman T, Porte RJ. Rebalanced hemostasis in patients with liver disease: evidence and clinical consequences. Blood 2010; 116: 878-85.

4. Henry Z, Northup PG. The rebalanced hemostasis system in end-stage liver disease and its impact on liver transplantation. Int Anesthesiol Clin 2017; 55: 107-20.

5. Lisman T, Leebeek FW, Mosnier LO, Bouma BN, Meijers JC, Janssen HL, et al. Thrombin-activatable fibrinolysis inhibitor deficiency in cirrhosis is not associated with increased plasma fibrinolysis. Gastroenterology 2001; 121: 131-9.

6. Weeder PD, Porte RJ, Lisman T. Hemostasis in liver disease: implications of new concepts for perioperative management. Transfus Med Rev 2014; 28: 107-13.

7. Ganter MT, Hofer CK. Coagulation monitoring: current techniques and clinical use of viscoelastic point-of-care coagulation devices. Anesth Analg 2008; 106: 1366-75.

8. Hartmann M, Szalai C, Saner FH. Hemostasis in liver transplantation: pathophysiology, monitoring, and treatment. World J Gastroenterol 2016; 22: 1541-50.

9. Kim TY. Perioperative bleeding disorder and intraoperative ponit-of-care testing of coagulation during cardiac surgery. Anesth Pain Med 2011; 6: 1-15.

10. Kaufman RM, Djulbegovic B, Gernsheimer T, Kleinman S, Tinmouth AT, Capocelli KE, et al. Platelet transfusion: a clinical practice guideline from the AABB. Ann Intern Med 2015; 162: 205-13. 